\title{
Lightning Low Level vs High Level Direct Current Injection Tests on a Full Scale Aircraft Cockpit
}

Fernando Cano Pérez

Guadalupe Gutierrez Gutierrez
Hugo Tavares

Abderrahim Khamlichi
Josefina Menendez Alberquilla

Raúl Molero Castejón
Nelson Matos

Angel Ramirez Linares

\begin{abstract}
This paper describes the Direct Current Injection (DCI) tests, in both Low and High levels, performed on a full scale aircraft cockpit with hybrid construction (carbon fiber composite and metallic parts). Additionally, some electromagnetic simulations carried out to optimize testing and analyze test results are also described.
\end{abstract}

\section{INTRODUCTION}

\section{A. Authors and Affiliations}

The activities described in the paper have been performed in the scope of the CaPAbilities for innovative Structural and functional teSting of AeROstructures (PASSARO) project (Clean Sky 2 Integrated Technology Demonstrators (ITD) Airframe) by a multidisciplinary team led by the PASSARO partner ISQ (Instituto de Soldadura e Qualidade), with important cooperation from Airbus DS and LCOE (Laboratorio Central Oficial de Electrotecnia) engineers and technicians. The activities are included in the Work Package 6 'Lightning strike assessment of panel structures and cockpit demonstrator' and Work Package 8 'Electromagnetic (EM) compatibility environment assessment of panels structures and full scale demonstrator'. More information related to the global targets of PASSARO project can be found in the corresponding web site [1].

\section{B. Objective}

Two different full scale tests have been performed:

- Low Level Direct Current Injection (LL-DCI) test has been performed at Airbus DS Getafe facilities (open Electro-Magnetic Compatibility (EMC) testing area).

- High Level Direct Current Injection (HL-DCI) test was performed at LCOE Getafe facilities (HL Lightning Strikes Laboratory).

The component under test was a full scale cockpit demonstrator approximately $4 \mathrm{~m}$ long, $2.9 \mathrm{~m}$ wide and $2.6 \mathrm{~m}$ high. The cockpit has hybrid construction based on the integration of Carbon Fiber Composite (CFC) and metallic components (see Fig. 1). It was equipped with two overbraided harnesses. Each harness had several branches ended at load boxes (a total of 7) grounded to the structure. The harnesses are filled with several inner conductors either with $50 \Omega$, short circuit or open circuit at their terminations so as to analyze different configurations.

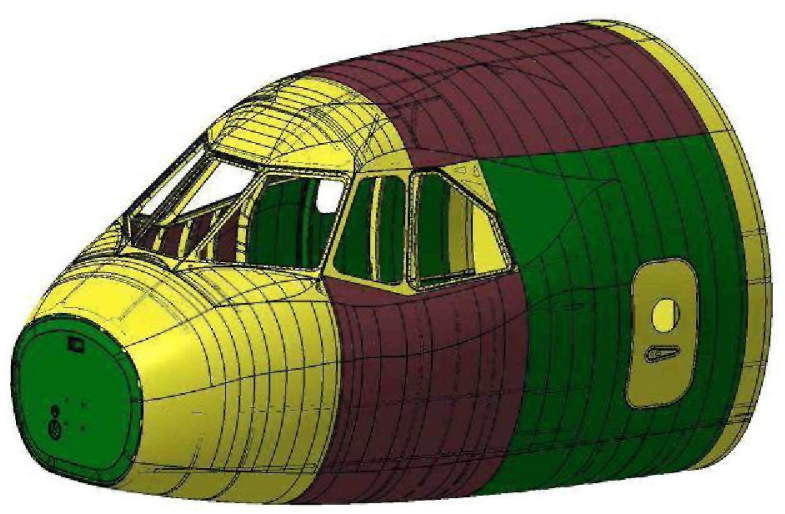

Fig. 1. Cockpit materials (Metal is in yellow CFC in green and CFC with Expanded Cooper Foil (ECF) in brown)

A model for EM simulations has been developed by ISQ using commercial software CST (Computer Simulation Technology). This model was used for evaluation/optimization of different test set-up options as well as to compare with corresponding test results. EM simulations are a very powerful tool to perform a practical and affordable assessment on the impact of changes to the airframe, systems, cable harnesses and materials.

\section{Low LEVEL DiRECT CURRENT INJECTION TEST}

\section{A. Test Method Description}

A LL-DCI test, also referred to as Low Level Direct Drive (LLDD), was carried out on the previously mentioned composite/metallic cockpit demonstrator at the Airbus Defence and Space Open Area Test Site (OATS). Test was performed in accordance with the corresponding standards [2][3].

The purpose of this test is to relate the currents induced into the cables due to an applied external field by relating, in a first step, these currents with the surface current densities excited in the aircraft skin by measurements, and, in a second step, the surface current densities excited in the aircraft skin with the applied external field by simulations. This technique is needed for a High-Intensity Radiated Field (HIRF) certification of an airframe from $10 \mathrm{kHz}$ up to $2 \mathrm{MHz}$ due to the impossibility of having a good radiating antenna at this lower frequency range, and its results are usable up to approximately the first airplane resonance. Several research studies have been published during the latest decades 
explaining the development of this method [4], [5], [6], [7], [8], [9], [10].

A cage return network intended to improve the transmission line characteristics of the drive network was built following the guidelines defined in section 6.1 .3 of [11] and taking into account the additional considerations described in [3], so that the cockpit structure plays the role of the core conductor.

A LL-DCI in time domain defined in [11] was also carried out in order to obtain data which can be easily compared with the HL-DCI performed later. To this end, not only the standard configuration in a LL-DCI with $50 \Omega$ at the exit, but also a short circuit at the exit was tested.

\section{B. Test set-up}

Fig. 2 shows the general arrangement of the test set-up with the wooden cradle supporting the cockpit and the coaxial return conductors. The cage coaxial return network consists of 40 copper conductors surrounding the cockpit from the injection to the exit points uniformly distributed and bonded in 4 transversal sections by means of aluminum bands, shaped to the form factor of the cabin along the length direction at each transversal section. In order to meet the requirements described in [3], a clearance of $45 \mathrm{~cm}$ from the cockpit skin to the 40 conductors was respected on all transversal planes, the largest distance between conductors was maintained within $30 \mathrm{~cm}$ and more than $25 \mathrm{~cm}$ of clearance exist between the floor and the bottom conductors of the return network.

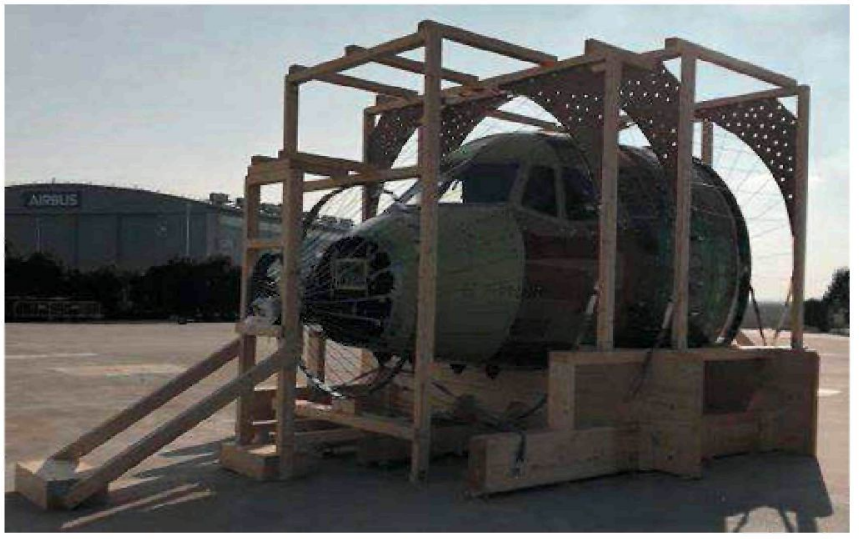

Fig. 2. LL DCI Test Set-Up - General view

The injection into the cabin skin was performed using 16 copper straps (see Fig. 3) and the exit by 1 centered copper strap with $50 \Omega$ at the termination. Injection and termination was made into the skin and coaxial return via high power coaxial connectors to line termination jigs, to adapt to the transmission lines used by the radio frequency (RF) instrumentation.

Before the start of the test campaign, bonding resistance between all relevant elements of the test setup (coaxial return network, injection and termination straps, termination boxes, over-braid harnesses, connectors, etc.) was performed to estimate the resistive voltage drop along the longitudinal direction of propagation.

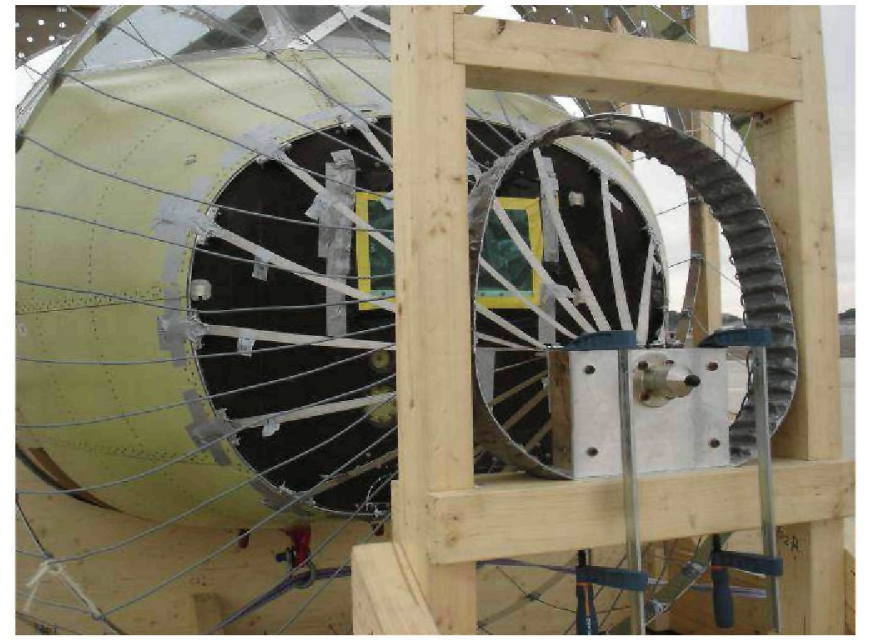

Fig. 3. LL DCI Test Set-Up - Injection area

The cockpit is equipped with two over-braid harnesses with several branches ending at terminal boxes. These harnesses allow the evaluation of the currents induced inside the interconnected instrumentation in different locations of the cabin via over-braided cabling. One harness is installed above the cabin floor; the second harness is installed below the cabin floor. The location of the terminal boxes is depicted and the over-braided route between simulated terminal equipment is illustrated on Fig. 4.

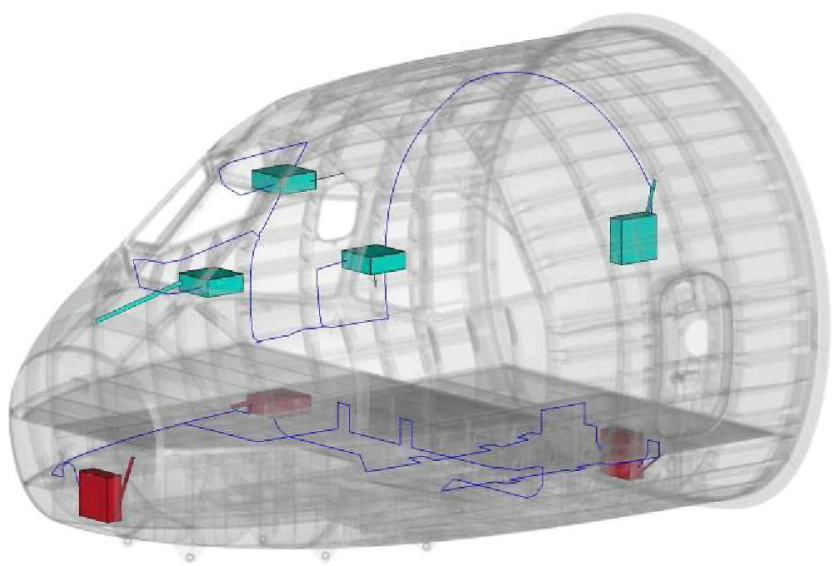

Fig. 4. Mockup of the layout of the instrumentation insidethe cabin.

The measured parameters, in magnitude and phase, were:

- The surface current density, or H-field, in both longitudinal and transversal propagation directions, at 16 points over the aerodynamic surfaces: 4 distributed along the top surface of the cockpit, 4 along the bottom part, 4 along the left side and 4 along the right side, with positions selected to sample on different materials of the cockpit skin (metal, CFC or CFC+ECF; see Fig. 1).

- The currents induced on the harness' over-braids at each branch (for ending branches, the probe was located at a distance of $50 \mathrm{~mm}$ from the harness end, while, for intermediate branches, the probe was located in the middle of the branches).

- The currents for $50 \Omega$ and short-circuit terminations, and voltages for open-circuit terminations, induced at each inner wire of the harnesses. These currents and voltages should be measured inside the shielded load boxes. 


\section{Test Procedure}

LL-DCI measurements in the frequency domain were performed using the test set-up shown in Fig. 5. The external generator a1 of a Vector Network Analyzer (VNA) was connected to the input of the RF amplifier. The injected current was measured by the VNA external port b1 and the desired current (surface current density, current induced on harnesses' over-braids or current/voltage coupled on inner conductors) by the VNA external port b2. Fiber optic links (FOL) were placed between current probes and VNA receivers in order to avoid cable losses and stray pickups. A normalized measurement $\mathrm{b} 2 / \mathrm{b} 1$ was performed by the VNA.

For LL Time Domain measurements, the RF instrumentation of Fig. 5 was replaced by a lightning indirect effects pulse generator. A waveform with a rise time of 6.4 $\mu$ s and a peak value of around $320 \mathrm{~A}$, named WF1 [12], was injected. The output of the pulse generator was connected to the injection point a coupler. The injected current was measured by the oscilloscope channel 1 and the desired current (surface current density or current induced on harnesses' over-braids) by the oscilloscope channel 2. As in the previous case, dedicated software performed all the necessary corrections.

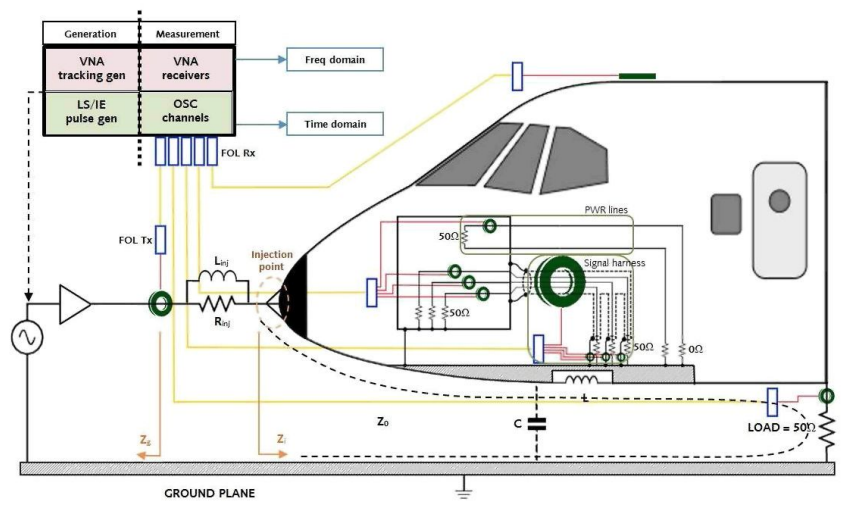

Fig. 5. LL DCI Test Set-Up - Measurements

\section{Test Results}

Fig. 6 shows the surface current density measured in the longitudinal direction at different positions in the cockpit skin in order to evaluate the surface current density distribution homogeneity obtained making use of the cage return network.

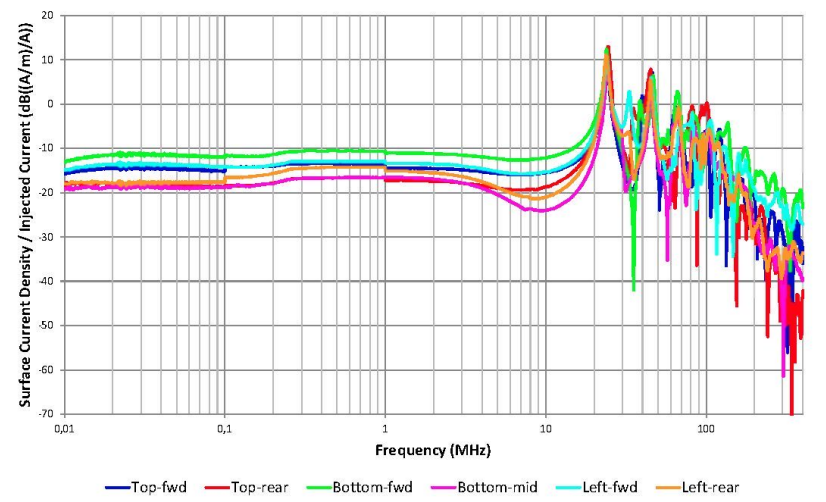

Fig. 6. LL DCI Test - Surface Current Density

The current induced in the over-braid of the load box 1 of top harness, along with the currents induced in its inner cables terminated locally in $50 \Omega$ and short circuit respectively are depicted in Fig. 7.

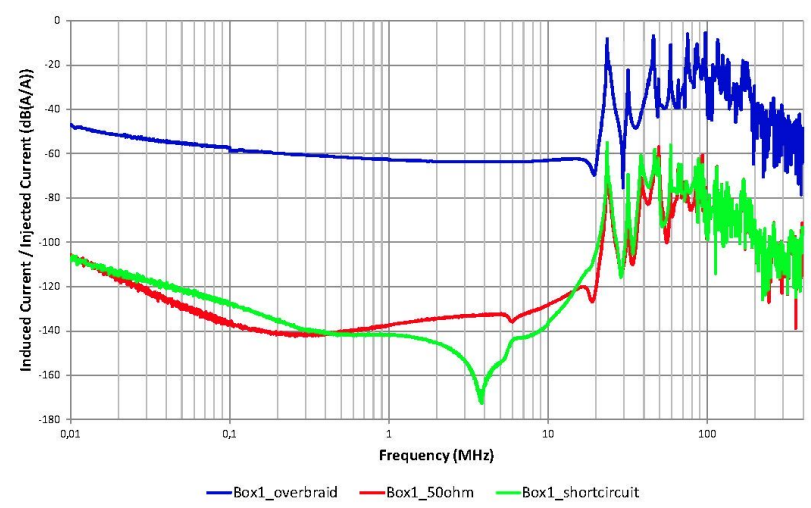

Fig. 7. LL DCI Test - Over-braid vs inner conductors induced currents

Fig. 8 shows the injected current in LL Time Domain measurements compared with the coupled current in the over-braid of the top harness at a distance of $50 \mathrm{~mm}$ from the load box 1 termination. This data was gathered with the termination of the cage network in short circuit.

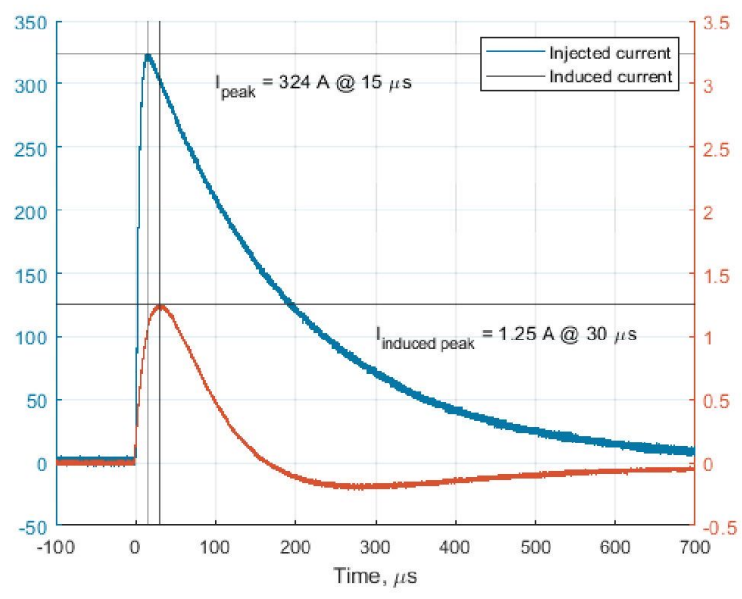

Fig. 8. LL DCI Test - Over-braid vs injected current

\section{E. Conclusions and Observations}

As it can be seen in Fig. 6, the homogeneity effect of the cage return network is good, especially in the lower part of the spectrum.

Shielding of the over-braided circuits show attenuations up to 50 to $60 \mathrm{~dB}$ in particular in the lower frequency range. While the over-braided circuits are bonded to the airframe, the inner circuits are terminated by $50 \Omega$ on at least one load box, which has contributed to the lower than expected level of the measured current relative to the specified shielding effectiveness of the over-braid. Fig. 7 shows that the wave shape between over-braid and inner circuit currents is very similar. This leads to believe that the most important mechanism of coupling inside the harness may be due to the structural current.

Comparing the time domain measurements illustrated on Fig. 8, a relationship between injected current to measured current peaks in the order of $50 \mathrm{~dB}$ is obtained.

As a lesson learnt for the future, the flatness of the test site floor must be taken into account in the design of the wooden cradle which supports the structure. Wooden wedges 
had ultimately to be fitted below the wooden cradle in order to level the structure and uniformly distribute the weight.

The weather conditions must also be taken into account. This testing campaign was performed at an OATS because not only DCI but the complete set of dedicated tests intended to demonstrate the immunity of aircraft equipment to HIRF defined in [2] and [3] were carried out, and it is not recommendable to perform some of these tests inside a hangar. However, the cold, rain and snow we had to face made this task difficult, slowed down the works and may have contributed to uncertainty between measurement batches.

\section{High LEVEL DIRECT CURRENT INJECTION}

\section{A. Test Method Description}

The HL-DCI consists in the injection of simulated lightning current pulses to the full scale component to evaluate the lightning current distribution (surface current densities) and the potential current/voltages coupled to the internal harnesses and equipment (termination boxes). Lightning current waveforms defined in [12] have been considered as a reference. The test has been performed at LCOE facilities in Getafe (High Current Generator) following the guidance material provided by [11], which specifies generally the test procedures to be taken. The objective of the test is:

- to verify the capabilities and limitations of performing a full scale component HL test;

- evaluate the results and compare them with those obtained in the Airbus DS facilities for a LL-DCI test;

- evaluate the effect on the homogeneity of the surface current density and coupled currents and voltages of two different coaxial return methods: 40 wire cage return used for LL-DCI versus 8 (or 4) solid copper bands for HL-DCI; and,

- compare LL and HL results with EM simulations.

\section{B. Test Set-up}

The specimen under test is the full scale equipped cockpit already used for LL-DCI tests. However, the supporting structure as well as the configuration for the return path and its configuration have been adapted to the specific HL test requirements (for instance, higher cross section conductors are required for lower frequency phenomena than that considered for LL-DCI) and taking into account the space constraints of the test laboratory (limited distance from floor to roof, so, very tight margin with cockpit dimensions) (see Fig. 9) as well as the non-ideal location of the test generators in respect to the to the cockpit. As a consequence of the limited height, both the clearance from the floor to the bottom copper bands and distance between copper bands and cabin skin had to be reduced to $20 \mathrm{~cm}$ and $25 \mathrm{~cm}$ respectively (minimum). Clearance to the ceiling was not has critical, having used a distance of about $50 \mathrm{~cm}$.

The rear aperture of the cockpit is closed by an aluminum cover that is also used for the adequate distribution of the current to the connected return path conductors (see Fig. 9).

The configuration for the current return network includes 8 copper bands distributed in order to conform to a quasicoaxial transmission line. Additional tests have been performed with only 4 conductors to evaluate the potential influence of a simplified return configuration.

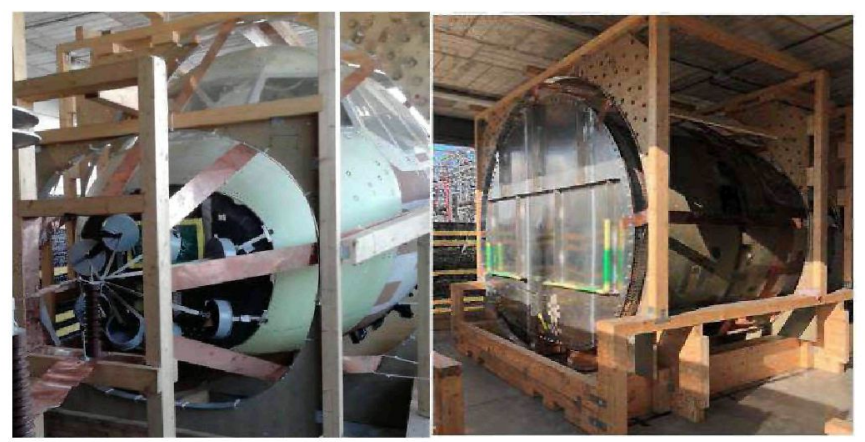

Fig. 9. HL DCI Test Set-Up - Injection (left); Rear cover (right)

The cockpit is equipped with two over-braid harnesses with several branches ending at terminal boxes. For the purposes of this test, one of the harnesses is fully monitored. Rogowski probes are placed at different branches to measure the coupled current into the over-braid; voltages at terminal boxes are recorded by an oscilloscope (see Fig. 10) on $50 \Omega$ and short circuit terminations. All the measuring system is autonomous and electrically isolated from the structure in order to avoid interferences.

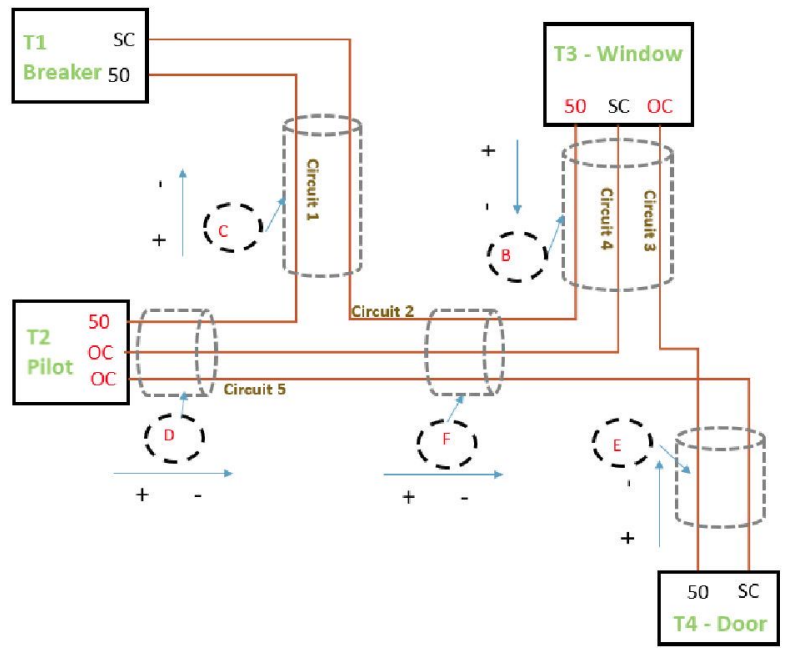

Fig. 10. HL DCI Test Set-Up - Top hamess measurements

\section{Test Procedure}

First step was to perform electrical bonding measurements to check all the installations (injection straps, return conductors, harnesses, inner circuits, connectors, boxes bonding, etc.) have been performed correctly. Due to the size of the Rogowski coils only 8 straps were used to inject lightning pulse into the cabin skin.

Subsequently, a set of current pulses were applied to the component under test in order to verify the correct lightning current distribution at the injection point, along the component and back through the return conductors.

Once the complete set-up was validated, four different current waveforms have been applied to the component in order to evaluate its response with regards to the different parameters (time to peak, amplitude, polarity, etc.) (see Fig. 11). 

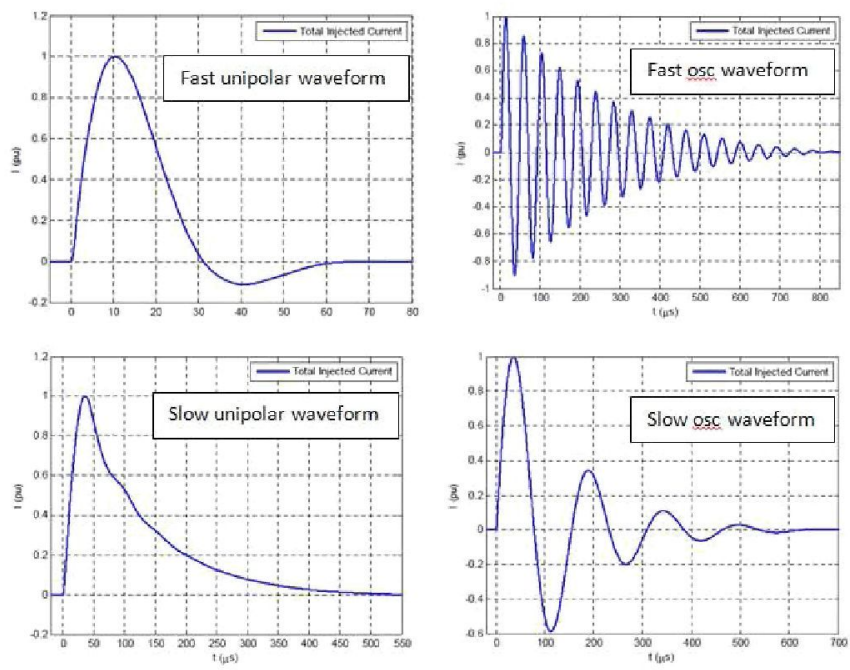

Fig. 11. HL DCI Test - Current waveforms injected

Several shots were performed at different levels $(10 \mathrm{kA}$, $50 \mathrm{kA}$ and $85 \mathrm{kA}$ ) to measure the surface current density in different points of the cockpit (CFC, $\mathrm{CFC}+\mathrm{ECF}$ and metallic areas) and with different probes.

A second series of shots at different levels (10 kA, 50 $\mathrm{kA}, 85 \mathrm{kA}$ and $125 \mathrm{kA}$ ) were performed to monitor the response of the internal harnesses and boxes terminations.

Additionally, two final shots were performed by arc injection in order to evaluate physical lightning direct effects damage. For this purpose, additional structural health monitoring systems were integrated in the cockpit in order to identify and evaluate the potential lightning damage in real time (description of these tests and systems are out of the scope of this paper, future dedicated paper will be focused on this matter). Additionally, it will be checked if the contact injection performed for measuring couplings has significant differences when the injection method is changed to are and the attaching point is laterally moved about one meter.

\section{Test Results}

The first series of tests consisted in measuring the surface current density at different points of the cockpit skin in order to evaluate the current distribution. The results are summarized in TABLE I. The resulting waveforms of the induced currents on the harnesses and boxes are summarised in TABLE II.

TABLE I. Surface CuRRent Densities Measurements

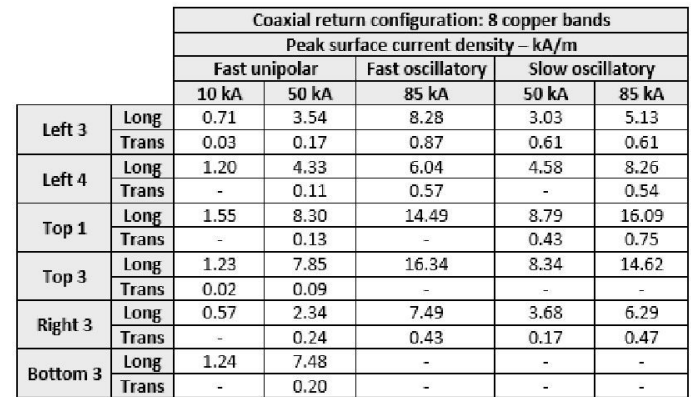

TABLE II. CURRENT AT HARNESS

\begin{tabular}{|c|c|c|c|c|c|c|c|c|c|c|c|}
\cline { 2 - 13 } \multicolumn{1}{c|}{} & \multicolumn{80}{c|}{ Coaxial return configuration: 8 copper bands } \\
\cline { 2 - 14 } \multicolumn{1}{c|}{} & Fast unipolar & \multicolumn{8}{c|}{ Fast oscillatory } & \multicolumn{7}{c|}{ Slow oscillatory } & \multicolumn{3}{c|}{ Slow unipolar } \\
\hline Level & 10 & 50 & 10 & 50 & 85 & 10 & 50 & 85 & 125 & 50 & 125 \\
\hline B & 0.09 & 0.38 & 0.13 & 0.40 & 0.66 & 0.13 & 0.68 & 1.23 & 1.823 & 0.69 & 1.84 \\
\hline C & 0.04 & 0.17 & 0.06 & 0.18 & 0.30 & 0.06 & 0.32 & 0.56 & 0.84 & 0.31 & 0.85 \\
\hline D & 0.06 & 0.22 & 0.073 & 0.22 & 0.36 & 0.07 & 0.37 & 0.61 & 0.87 & 0.37 & 0.89 \\
\hline E & 0.002 & 0.005 & 0.003 & 0.01 & 0.02 & 0.004 & 0.02 & 0.03 & 0.59 & 0.01 & 0.13 \\
\hline F & 0.09 & 0.37 & 0.12 & 0.36 & 0.59 & 0.13 & 0.65 & 1.05 & 1.56 & 0.66 & 1.59 \\
\hline Injected & 11.7 & 48.91 & 15.93 & 49.79 & 84.32 & 9.71 & 49.77 & 84.55 & 125.44 & 49.84 & 125.67 \\
\hline
\end{tabular}

\begin{tabular}{|c|c|c|c|c|c|c|c|}
\cline { 2 - 7 } \multicolumn{1}{c|}{} & \multicolumn{6}{c|}{ Coaxial return configuration: 8 copper bands } & Coaxial return configuration: 4 copper bands \\
\cline { 2 - 7 } \multicolumn{1}{c|}{} & \multicolumn{6}{c|}{ Slow oscillatory } & \multicolumn{3}{c|}{ Arc strikes } \\
\cline { 2 - 7 } & \multicolumn{7}{|c|}{ Peak induced current - kA } \\
\hline Level & $\mathbf{1 0}$ & 50 & $\mathbf{8 5}$ & $\mathbf{1 2 5}$ & $\mathbf{5 0}$ & $\mathbf{1 1 0}$ \\
\hline B & 0.13 & 0.68 & 1.23 & 1.82 & 0.81 & 2.13 \\
\hline C & 0.06 & 0.32 & 0.56 & 0.84 & 0.35 & 0.86 \\
\hline D & 0.07 & 0.37 & 0.61 & 0.87 & 0.44 & 1.01 \\
\hline E & 0.004 & 0.020 & 0.033 & 0.059 & 0.026 & 0.070 \\
\hline F & 0.13 & 0.652 & 1.05 & 1.56 & 0.69 & 1.57 \\
\hline Injected & 9.71 & 49.77 & 84.55 & 125.44 & 45 & 112 \\
\hline
\end{tabular}

\section{E. Conclusions and Observations}

From this test, several conclusions and observations can be extracted:

- The performance of a complete full scale component HL test is a very complex activity. Not only pure technical aspects are critical drivers to be considered in order to perform a satisfactory test but also logistic aspects (such as the handling of the component and assembly of all the supporting structure and return path). Physical constrains (for instance, the distance from the generator to the component due to laboratory dimensional constrains increase the global inductance of the circuit and affects the current waveform that can be achieved - both in rise time and peak amplitude) should be carefully analyzed in advance in order to estimate the possible impact on the ideal injected current waveform on the component;

- In accordance with the previous observations, currents and voltages measured at termination boxes are also higher when low frequency content waveforms are injected;

- Current observed at transversal harness is very low compared with the other harnesses that are aligned with the current path;

- It is confirmed that the change from 8 to 4 copper bands in the return conductors affects the current distribution in the cockpit. As expected, higher number of return conductors implies more homogenous current density in the test specimen.

\section{Electromagnetic Simulations}

\section{A. Model Description}

A model of the test setup has been generated by using comercial software package CST. This model is used for EM simulations in order to compare with the results from both the LL and HL test campaigns. To evaluate LL couplings, the cage return of Fig. 2 was used as illustrated in Fig. 12. On this model, a physical equivalent of the instrumentation fitted into the cabin with equivalent loads was implemented (boxes, straps, etc). 


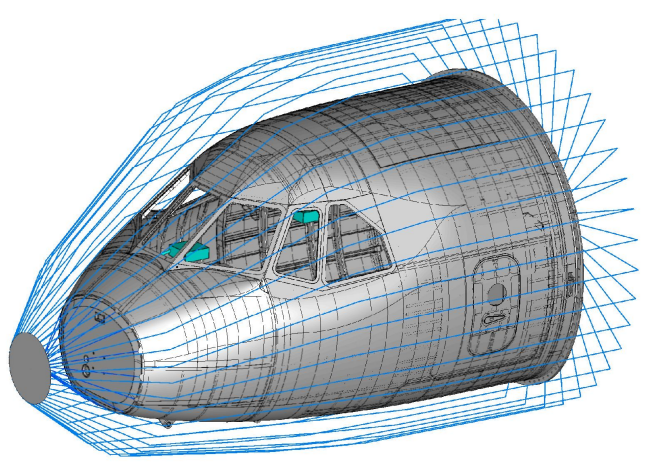

Fig. 12. DCI-LL Simulation model.

The coaxial return is modeled as solid wires. A discrete port in the nose is used to excite the structure and extract the Sparameters. Post-processing routines are implemented to compute the normalized surface currents and induced loom currents. A comparison of simulated and measured surface current densities and harness currents is shown on Fig. 13 and Fig. 14 respectively.

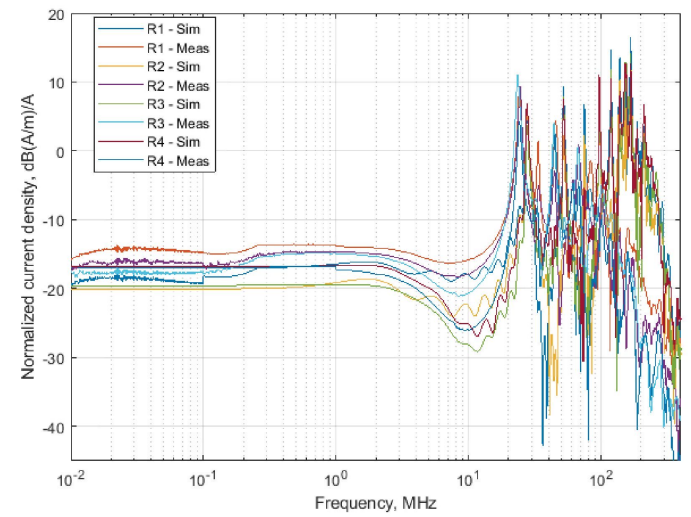

Fig. 13. Normalized surface current density - Simulation vs Measurement.

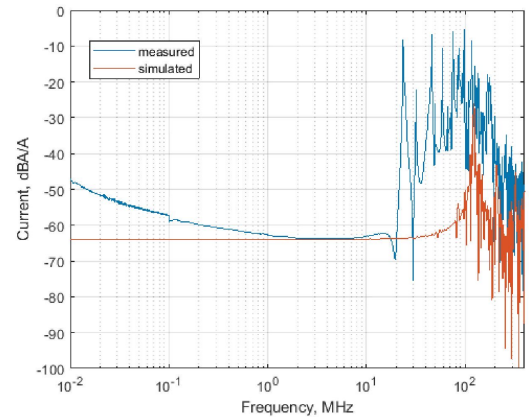

Fig. 14. Top harness overbraid currents - Simulation vs Meas urement.

\section{B. Conclusions and Observations}

Modeling and simulation of such a large specimen is a complex task. Accurary must be balanced with computation power to obtain representative results. The data already obtained for LL simulations show a good agreement with the measured data. In particular, normalized surface current densities seem to correlate well up to $100 \mathrm{MHz}$. Above 100 $\mathrm{MHz}$, the data shows some deviations. Sensitivity analysis has shown how critical the meshing of the structure and the position of the point of interest can influence the results. Regarding induced currents into the looms, the comparison seems to show that in low frequency the measurement system sensitivity seems low, since at low frequency a low induced current level is expected, due to the small electrical size at these frequencies. The simulated currents do not correlate especially between $10 \mathrm{MHz}$ and $100 \mathrm{MHz}$.

\section{Final Conclusions}

A comparison between a physical large specimen for DCILL and DCI-HL measurements has been performed (see agreement in TABLE III). It was shown the importance of the coaxial return and that the use of commercially available tools for EM simulation can greatly help in the preparation and optimization of large scale test setup campaigns.

TABLE III. DCI-LL VS DCI-HL CURRENTS INDUCED ON LOOMS

\begin{tabular}{|c|c|c|c|c|}
\cline { 2 - 5 } \multicolumn{1}{c|}{} & \multicolumn{2}{c|}{$\mathrm{DCl}-\mathrm{LL}$ (peak time $6 \mu \mathrm{s})$} & \multicolumn{2}{c|}{$\mathrm{DCl}-\mathrm{HL}$ (peak time $10 \mu \mathrm{s})$} \\
\cline { 2 - 5 } \multicolumn{1}{c|}{} & $\mathrm{I}_{\text {ini }}=328 \mathrm{~A}$ & $\mathrm{I}_{\text {ind }} / \mathrm{I}_{\text {ini }}(\%)$ & $\mathrm{I}_{\text {ini }}=50 \mathrm{kA}$ & $\left.\mathrm{I}_{\text {ind }} / \mathrm{I}_{\text {ini }} \%\right)$ \\
\hline $\mathrm{I}_{\text {induced }}$ On LTB1 & $1,26 \mathrm{~A}$ & $0.38 \%$ & $171 \mathrm{~A}$ & $0.34 \%$ \\
\hline $\mathrm{I}_{\text {induced }}$ on LTB2 & $1,92 \mathrm{~A}$ & $0.59 \%$ & $223 \mathrm{~A}$ & $0.45 \%$ \\
\hline $\mathrm{I}_{\text {induced }}$ on LTB3 & $3 \mathrm{~A}$ & $0.91 \%$ & $384 \mathrm{~A}$ & $0.77 \%$ \\
\hline $\mathrm{I}_{\text {induced }}$ on LTB4 & $0,12 \mathrm{~A}$ & $0.037 \%$ & $5 \mathrm{~A}$ & $0.01 \%$ \\
\hline
\end{tabular}

ACKNOWLEDGMENT

The work described in this paper has received funding from the European Community's H2020-EU.3.4.5.4. - ITD Airframe Programme with Topic CS2-GAM-2018-AIR Airframes under grant agreement ID 807083. 